\title{
Photogeneration of membrane potential hyperpolarization and depolarization in non-excitable cells
}

\author{
Jun Ando • Nicholas I. Smith · Katsumasa Fujita • \\ Satoshi Kawata
}

Received: 12 June 2008 / Revised: 2 December 2008 / Accepted: 14 December 2008 / Published online: 9 January 2009

(C) The Author(s) 2009. This article is published with open access at Springerlink.com

\begin{abstract}
We monitored femtosecond laser induced membrane potential changes in non-excitable cells using patchclamp analysis. Membrane potential hyperpolarization of HeLa cells was evoked by $780 \mathrm{~nm}, 80$ fs laser pulses focused in the cellular cytoplasm at average powers of 30$60 \mathrm{~mW}$. Simultaneous detection of intracellular $\mathrm{Ca}^{2+}$ concentration and membrane potential revealed coincident photogeneration of $\mathrm{Ca}^{2+}$ waves and membrane potential hyperpolarization. By using non-excitable cells, the cell dynamics are slow enough that we can calculate the membrane potential using the steady-state approximation for ion gradients and permeabilities, as formulated in the GHK equations. The calculations predict hyperpolarization that matches the experimental measurements and indicates that the cellular response to laser irradiation is biological, and occurs via laser triggered $\mathrm{Ca}^{2+}$ which acts on $\mathrm{Ca}^{2+}$ activated $\mathrm{K}^{+}$channels, causing hyperpolarization. Furthermore, by irradiating the cellular plasma membrane, we observed membrane potential depolarization in combination with a drop in membrane resistance that was consistent with a transient laser-induced membrane perforation. These results entail the first quantitative analysis of locationdependent laser-induced membrane potential modification and will help to clarify cellular biological responses under exposure to high intensity ultrashort laser pulses.
\end{abstract}

J. Ando $(\bowtie) \cdot$ N. I. Smith $\cdot$ K. Fujita $\cdot$ S. Kawata

Department of Applied Physics, Osaka University,

2-1 Yamadaoka, Suita, Osaka 565-0871, Japan

e-mail: ando@ap.eng.osaka-u.ac.jp

N. I. Smith

Photonics Advanced Research Center, Osaka University,

2-1 Yamadaoka, Suita, Osaka 565-0871, Japan
Keywords Femtosecond laser - Membrane potential . Laser-cell interaction · Hyperpolarization · Depolarization

\author{
Abbreviations \\ $\left[\mathrm{Ca}^{2+}\right]_{i} \quad$ Intracellular calcium ion concentration \\ GHK voltage Goldman-Hodgkin-Katz equation \\ equation
}

\section{Introduction}

The use of the near-infrared femtosecond laser has become popular for biomedical experiments, due to the long penetration depth, low scattering, and localized nonlinear absorption (Denk et al. 1990; Dombeck et al. 2005; Smith et al. 2001; König et al. 1999; Hirase et al. 2002). These advantages have been used to carry out novel experiments in cells such as generation of intracellular $\mathrm{Ca}^{2+}$ waves (Smith et al. 2001), dissection of intracellular chromosomes (König et al. 1999), as well as inducing action potentials in neurons (Hirase et al. 2002). While similar effects have been produced using different laser sources (Uzdensky and Savransky 1997; Wells et al. 2007), the merits of femtosecond laser irradiations are being seen in the emergence of new applications which exploit the particular properties of ultrashort pulsewidths and near-infrared wavelengths. Although such applications are now emerging, the use of femtosecond laser illumination to provoke biological reactions in cells is in part limited by the incomplete understanding of the cellular response to high intensity light. There is therefore significant interest in finding new ways to understand and measure the cell response to high intensity light, and particularly the response to femtosecond laser irradiation.

In this report, we use patch-clamp measurement to monitor cellular membrane potential during femtosecond laser 
irradiation. Patch-clamp recording allows quantitative evaluation of the laser effect on the cell membrane condition. HeLa cells were chosen specifically as targets for their lack of action potentials and slow electrophysiological response, allowing us to determine the laser effect on the cell electrophysiology. Changes in membrane potential following laser irradiation were monitored, and clear differences in membrane potential response were observed depending on the location of the laser focus.

\section{Experimental}

To perform the experiments, we used a set-up shown in Fig. 1. This set-up is the same as previously reported by Smith et al. (2001), with the exception that an inverted fluorescence microscope (IX-70, Olympus) was used with a higher numerical aperture (NA) water immersion objective lens $(60 \times / \mathrm{NA} 1.0$, Olympus $)$. The femtosecond laser (Tsunami, Spectra Physics) irradiation parameters were: average power of $10-60 \mathrm{~mW}$, repetition rate of $82 \mathrm{MHz}$, wavelength centered at $780 \mathrm{~nm}$, pulsewidth of $80 \mathrm{fs}$ at emission. Average laser power is used as an overall parameter to gauge the strength of laser effects throughout this manuscript. In order to compare to other experiments, at an average laser power of $10 \mathrm{~mW}$ for the femtosecond pulsed mode-locked irradiation, the pulse energy is $0.122 \mathrm{~nJ}$, peak power is $813 \mathrm{~W}$, and if the diffraction limited spot diameter is $497 \mathrm{~nm}$, and the pulse disperses to approximately $170 \mathrm{fs}$ during propagation through the optics, the peak intensity at the sample is then $0.420 \mathrm{TW} / \mathrm{cm}^{2}$. These values scale linearly with average power. The cells used in this procedure

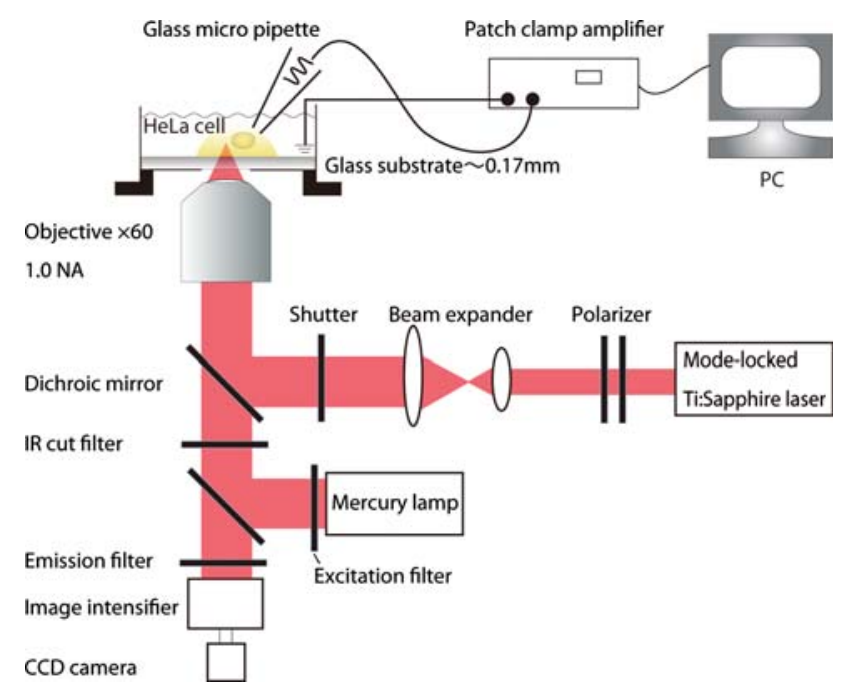

Fig. 1 Experimental set-up for the simultaneous measurement of photoinduced membrane potential and $\mathrm{Ca}^{2+}$ dynamics were HeLa (cancerous epithelial) cells, grown in the same procedures as reported by Iwanaga et al. (2006). For the electrophysiological measurement, cells were immersed in a bath solution containing $\mathrm{NaCl}(116 \mathrm{mM}), \mathrm{KCl}(5.4 \mathrm{mM})$, $\mathrm{CaCl}_{2}(1.8 \mathrm{mM})$, HEPES $(25 \mathrm{mM}), \mathrm{NaH}_{2} \mathrm{PO}_{4}(1.0 \mathrm{mM})$, Glucose $(5.5 \mathrm{mM}), \quad \mathrm{NaHCO}_{3} \quad(6 \mathrm{mM}), \quad$ and $\mathrm{MgSO}_{4}$ $(0.8 \mathrm{mM})$ at $\mathrm{pH}=7.4$ (Roy and Sauvé 1983). This solution was remade before each experiment, and the $\mathrm{pH}$ value was confirmed to be 7.4 before starting the experiments. Patch pipettes were filled with solution containing $150 \mathrm{mM} \mathrm{KCl}$, and $3 \mathrm{mM}$ HEPES at $\mathrm{pH}=7.2$. Electrical measurement was performed with a patch clamp amplifier (Axopatch 200B, Molecular Devices), using patch pipettes fabricated from borosilicate glass capillaries with an outside diameter of $1.5 \mathrm{~mm}$ (TW150-3, World Precision Instrument) using a vertical pipette puller (pp-830 puller, Narishige). Fabricated patch pipettes were manipulated by hydraulic type manipulator (MHW-3, Narishige). Target cells were sealed by a patch pipette to form an electrical seal resistance of at least several G $\Omega$. Then suction was applied to break the attached membrane at the pipette apex, and complete the whole-cell patch configuration. With the cell and micropipette held in whole-cell patch mode, the cell was exposed to laser irradiation, at one of two locations. In the case where the cytoplasm was targeted, the laser focus was positioned at a distance of approximately $2 \mu \mathrm{m}$ outside the nucleus and $2 \mu \mathrm{m}$ below the cell membrane. The laser focus height was adjusted by observing the multiphoton fluorescence from the cytoplasm. For irradiation of the cell membrane, the laser focus was positioned at the outer edge of the cell. Exposure time was limited to $8 \mathrm{~ms}$ by a mechanical shutter and the mean laser power was set to $20-60 \mathrm{~mW}$, as measured at the focus by a thermal power meter. For the measurement of $\mathrm{Ca}^{2+}$ dynamics, the cells were loaded with Fluo-4 by exposing them to Fluo-4 ester in bath solution (0.018 mM, Invitrogen) for $30 \mathrm{~min}$ at room temperature. Subsequently, dye remaining in the extracellular solution was removed by washing with fresh bath solution. $\mathrm{Ca}^{2+}$ dependent fluorescence was observed using a mercury lamp illumination source and intensified charge-coupled-device camera (C2400-35, Hamamatsu) for imaging. HeLa cells which we used in this study showed an overall average resting potential of $-17.0 \mathrm{mV}$ out of 37 cells, with a standard deviation of 12.4. Due to the time-consuming nature of the measurements, gathering data required the combination of measurements from target cells in different culture dishes. Cells were used on the second day after seeding, and the $\mathrm{pH}$ was tested before each experiment, as outlined above. The resting potentials of individual cells were observed to vary between cells and dishes, but for all data that was used for analysis, the resting potentials prior to laser irradiation lay within the range of resting potentials exhibited by HeLa cells (Borle and Loveday 1968). 
Fig. 2 a Femtosecond laser induced membrane potential hyperpolarization of HeLa cells following laser irradiation of cellular cytoplasm. b Hyperpolarization depth dependence on laser power. c Recovery time dependence on laser power. We defined the recovery time as the period of time from the laser irradiation to the time when the membrane potential recovered to within $\pm 5 \%$ of the initial resting potential. d Hyperpolarizability probability dependence on laser power and, for comparison, the $\mathrm{Ca}^{2+}$ wave generation probability dependence on laser power (Iwanaga et al. 2006)
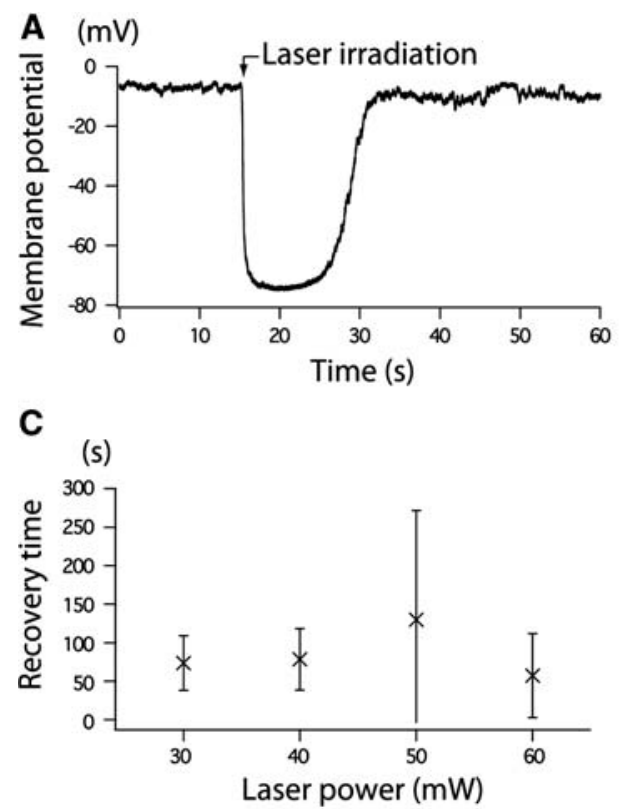

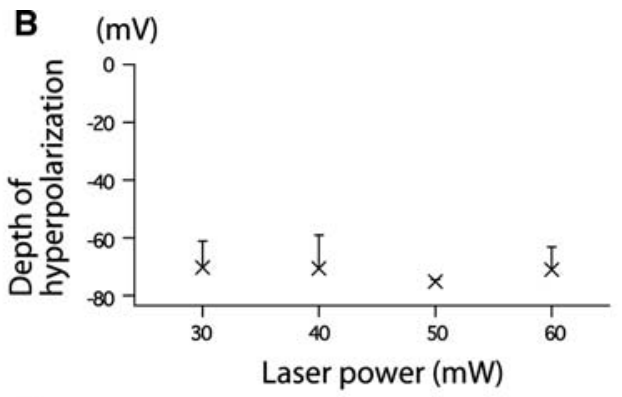

D

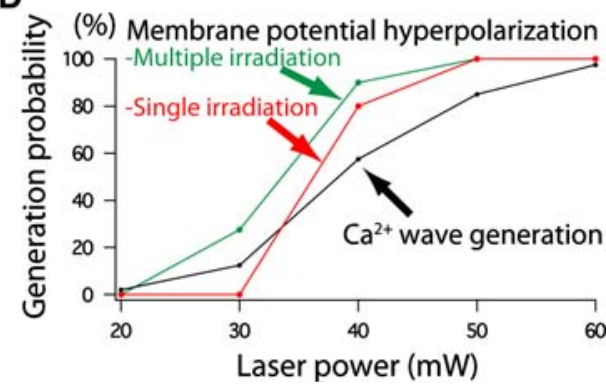

\section{Results and discussion}

Figure 2a shows femtosecond laser induced membrane potential hyperpolarization of HeLa cells for irradiation focused in the cellular cytoplasm, with an average power of $60 \mathrm{~mW}$ and an exposure time of $8 \mathrm{~ms}$. From 21 data sets, the pre-irradiation average resting membrane potential was $-12.1 \mathrm{mV}$ with a standard deviation of 6.0. Following laser irradiation, the membrane potential of HeLa cell exhibited rapid hyperpolarization. Almost all cells (18 out of 21) showed an apparently saturated and stable hyperpolarization at around $-74.9 \mathrm{mV}$ shown in Fig. 2b. Hyperpolarization duration was between several seconds to several hundred seconds, with a subsequent gradual return to the initial resting potential. The variation in the period between laser irradiation and the return to the initial resting potential is shown in Fig. 2c. We also measured the hyperpolarization dependence on laser power and examined the effects of multiple exposures. Cells are first irradiated by a single $8 \mathrm{~ms}$ exposure and where no response was observed, the exposure was repeated up to ten times at intervals of $5 \mathrm{~s}$. From this data, we could extract the single vs. multiple exposure power dependence, as shown in Fig. 2d, where each data points on the graph shows the hyperpolarization response of 5 independent cells. The onset of laser-induced membrane potential hyperpolarization can be seen to occur at around $30 \mathrm{~mW}$ of laser power. The behaviour and onset of the hyperpolarization power dependence is very similar to that of laser induced $\mathrm{Ca}^{2+}$ wave generation, which is included on the graph for comparison.

To consider the nature of the photoinduced membrane potential change, we also monitored the membrane potential of HeLa cells under the influence of continuous-wave $780 \mathrm{~nm}$ irradiation, and no response in membrane potential or $\mathrm{Ca}^{2+}$ concentration was observed even with $60 \mathrm{~mW}$ of laser power, while femtosecond pulsed $780 \mathrm{~nm}$ laser with $60 \mathrm{~mW}$ of laser power causes apparent membrane potential hyperpolarization. This result is similar to results reported by Sacconi et al., where continuous wave near-infrared laser irradiation was not able to ablate microtubules, while femtosecond pulsed laser of the same power could cause ablation (Sacconi et al. 2005). The requirement of the ultrashort pulsed mode indicates that the nature of the photostimulation of the membrane potential hyperpolarization is clearly nonlinear, and is likely related to multiphoton-based ionization, in agreement with previous work (Iwanaga et al. 2006; Vogel and Venugopalan 2003). The requirement for the femtosecond pulsed mode occurs due to the low linear absorption of photons at $780 \mathrm{~nm}$, and a less stringent requirement for pulsed irradiation would be expected if the wavelength was reduced. However, $780 \mathrm{~nm}$ is ideal for irradiation of living biological targets due to the high penetration and low absorption in areas outside the focal region (Denk et al. 1990). Previous work using the same cell type showed that, depending on the pulse width and repetition rate, the interaction between near-infrared ultrashort pulsed irradiation and the resulting cell response can even become independent of the exposure time, showing the highly nonlinear nature of the interaction (Iwanage et al. 2005). The observation of such $\mathrm{Ca}^{2+}$ responses in living cells from even a single pulse of low pulse energy further supports the premise that the interaction is based on the nonlinear interactions resulting from the ultrashort pulsewidth rather than single photon, thermal, or other mediating processes in the laser-cell interaction.

The apparent relationship shown in Fig. 2d between photoinduced membrane potential hyperpolarization and $\mathrm{Ca}^{2+}$ wave generation was further investigated. Femtosecond 
laser illumination causing $\mathrm{Ca}^{2+}$ wave generation has been reported in HeLa cells (Smith et al. 2001; Iwanaga et al. 2006), and can potentially affect membrane voltage (Roy and Sauvé 1983; Tilly et al. 1990). The nature and properties of the laser-induced $\mathrm{Ca}^{2+}$ response were analyzed in a previous study (Iwanaga et al. 2006). The conclusion of that study was that for irradiation of the cytoplasm, the intracellular calcium stores, which are spread through the cytosol, are induced to release a trigger amount of $\mathrm{Ca}^{2+}$ which can then be self-amplified by $\mathrm{Ca}^{2+}$ induced $\mathrm{Ca}^{2+}$ release, leading to a significant intracellular $\mathrm{Ca}^{2+}$ elevation over the entire cell from the laser interaction at the focal region. To analyze the relation of intracellular ion dynamics and membrane potential change, photoinduced changes in membrane potential and intracellular $\mathrm{Ca}^{2+}$ concentration $\left(\left[\mathrm{Ca}^{2+}\right]_{i}\right)$ were observed simultaneously. Following laser irradiation, membrane potential hyperpolarization and $\left[\mathrm{Ca}^{2+}\right]_{i}$ elevation occurred simultaneously, as shown in Fig. 3. The hyperpolarization and $\left[\mathrm{Ca}^{2+}\right]_{i}$ wave duration were also similar in length. The strong correlation between the two measurements indicates a causal relationship, and both quantities can conceivably affect each other. We can, however, predict the membrane potential from the known quantities of intracellular ions, gradients and cell permeabilities. In addition, HeLa cells' membrane potassium channels are known to be nonlinearly and highly sensitive to calcium concentrations, and undergo a 100 -fold increase in permeability in response to $\left[\mathrm{Ca}^{2+}\right]_{i}$ elevation (Sauvé et al. 1986). Using the Goldman-Hodgkin-Katz (GHK) voltage equation, we can calculate the predicted membrane voltage before and after the photoinduced $\mathrm{Ca}^{2+}$ concentration eleva- tion. By the GHK calculation using known parameters for HeLa cells (Wickson-Ginzburg and Solomon 1981; Wehner et al. 2003), the predicted initial membrane potential was $-9.4 \mathrm{mV}$, while the predicted potential following laser-induced $\mathrm{Ca}^{2+}$ elevation was $-82.2 \mathrm{mV}$. These correspond well with the average measured values of -12.1 and $-74.9 \mathrm{mV}$, respectively. The predictions are based on the known property (Sauvé et al. 1986) that $\mathrm{Ca}^{2+}$ sensitive $\mathrm{K}^{+}$ channel permeability undergoes a 100 -fold increase, with $\left[\mathrm{K}^{+}\right]_{i}=170 \mathrm{mM},\left[\mathrm{Na}^{+}\right]_{i}=32 \mathrm{mM}$ and $\left[\mathrm{Cl}^{-}\right]_{i}=43 \mathrm{mM}$ and

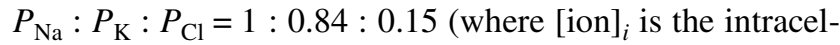
lular ion concentration, and $P_{x}$ is the permeability of ion ' $x$ ').

We then lowered the extracellular potassium concentration from $5.4 \mathrm{mM}$ to $0.4 \mathrm{mM}$ and repeated the experiment. In this case, the average depth of membrane potential hyperpolarization changed to $-111.5 \mathrm{mV}(n=5)$. This value again corresponds well with the prediction of $-114.9 \mathrm{mV}$, as calculated by the GHK equation with the appropriate modification for the lowered $\mathrm{K}^{+}$concentration. This indicates that the femtosecond laser induced membrane potential hyperpolarization was caused by rapid elevation of the $\mathrm{K}^{+}$channels open probability, which was in turn triggered by laser-induced $\left[\mathrm{Ca}^{2+}\right]_{i}$ elevation.

The cytoplasm irradiation experiments caused hyperpolarization that was consistent with known cellular properties. We also performed similar experiments, instead targeting the outer cell membrane, where we expected to see significantly different responses in membrane potential due to the disruption of the membrane. Evidence of membrane disruption after membrane irradiation by femtosecond
Fig. 3 Coincident photogeneration of membrane potential hyperpolarization and $\mathrm{Ca}^{2+}$ waves following laser irradiation of the cytoplasm. Upper figures show a time sequence of fluorescence images of HeLa cells stained with the $\mathrm{Ca}^{2+}$ indicator Fluo-4. (White dot shows laser focus, white line shows pipette apex, and white dotted circle shows targeted cell.) Lower figure shows femtosecond laser causing membrane potential hyperpolarization and intracellular $\mathrm{Ca}^{2+}$ concentration elevation in a HeLa cell. The scale bar shows $20 \mu \mathrm{m}$

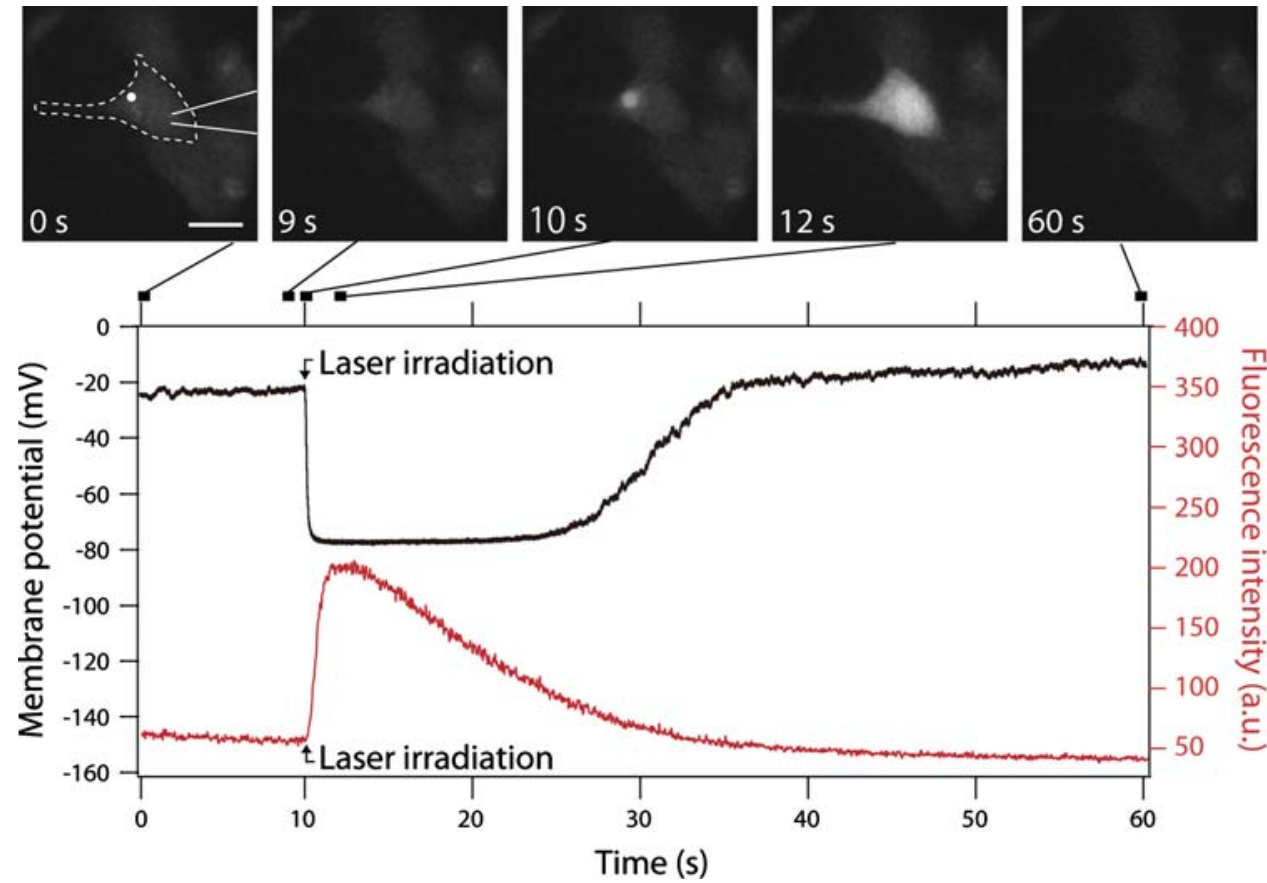


laser has been previously seen, where influx of $\mathrm{Ca}^{2+}$ or other extracellular molecules could occur and affect the intracellular $\mathrm{Ca}^{2+}$ response (Iwanaga et al. 2006), as well as by other experiments where influx of extracellular molecules was intentionally induced in order to modify the cell (Tirlapur and König 2002). It should be noted, however, that the laser disruption of the outer cell membrane precludes the use of the GHK equations to calculate the membrane potential, as was done in the case of cytosol irradiation, since the ion-specific permeability after irradiation is unknown and the ion flow across the membrane cannot be approximated as steady-state.

Figure 4 shows membrane potential depolarization by laser irradiation of the membrane. The laser power was $60 \mathrm{~mW}$, and the membrane was exposed for $8 \mathrm{~ms}$. From 16 data sets, the pre-irradiation average resting membrane potential was $-23.4 \mathrm{mV}$ with a standard deviation of 15.3. Following laser irradiation, the membrane potential exhibited rapid depolarization to reach an averaged peak potential of $+3.7 \mathrm{mV}(n=16)$ in a timeframe within $50 \mathrm{~ms}$ following laser irradiation. After depolarization, 7 out of 16 cells showed membrane potential repolarization (examples of which are shown in Fig. 4a), while the remaining 9 cells showed a stable potential at around $0 \mathrm{mV}$ (shown in Fig. 4b). We also measured the membrane resistance before and after the laser illumination on cellular plasma membrane, and observed a significant decrease. In cases where the membrane potential repolarized after depolarization, the average resistance decreased from 629.0 to $337.5 \mathrm{M} \Omega$ $(n=5)$. When, however, the depolarized potential remained stable at around $0 \mathrm{mV}$, the average resistance decreased from 567.2 to $12.9 \mathrm{M} \Omega(n=7)$. The marked difference in post-irradiation resistance for cells that did not repolarize indicates a non-sealing physical perforation of the cellular plasma membrane by laser illumination. In 2005, Fein et al. also observed membrane potential depolarization and resistance decrease following femtosecond laser disruption of the cellular plasma membrane (Fein and Terasaki 2005). More recently, a study of membrane potential changes by thermal effects produced by mid-infrared photons showed that thermal effects can affect the membrane potential if the temperature rise is more than a few degrees (Wells et al. 2007). Previous calculations for our optical system (Iwanaga et al. 2006) which estimated a temperature rise of less than 1 degree, together with the large drop in observed membrane resistance indicates that the effect of the laser is to create a transient hole in the membrane. Further evidence for the existence of a physical perforation was observed in a previous report where penetration of EGTA from the extracellular solution was observed in cultured HeLa cells following irradiation of the cell membrane with the same optical conditions (Iwanaga et al. 2006). Additional information regarding the possible existence of a membrane perforation can be gained by comparing the time course of the membrane potential recovery for cytoplasm irradiation and membrane irradiation, respectively. Figure 5 shows the time course of the membrane potential following membrane irradiation (Fig. 5a) and following cytoplasm irradiation (Fig. 5b), and for comparison, shows a longer time course of the membrane potential following cytoplasm and membrane irradiation (Fig. 5c). From the comparison of these results, the membrane potential following membrane irradiation shows more unstable behavior after laser irradiation. Therefore, we presume that $60 \mathrm{~mW}$ of laser power for plasma membrane irradiation can be difficult for the cell to completely reseal, and thus causes the observed unstable behavior. To discuss the mechanism underlying the membrane potential repolarization after depolarization following membrane irradiation, we also performed the simultaneous measurement of membrane potential and $\mathrm{Ca}^{2+}$ dynamics shown in Fig. 6. Following membrane irradiation, both membrane potential depolarization and intracellular $\mathrm{Ca}^{2+}$ concentration elevation were observed, and the membrane potential showed rapid repolarization after
Fig. 4 Femtosecond laser induced membrane potential depolarization of HeLa cells following laser irradiation of cellular plasma membrane. a Repolarization after depolarization. b Showing $0 \mathrm{mV}$ after depolarization
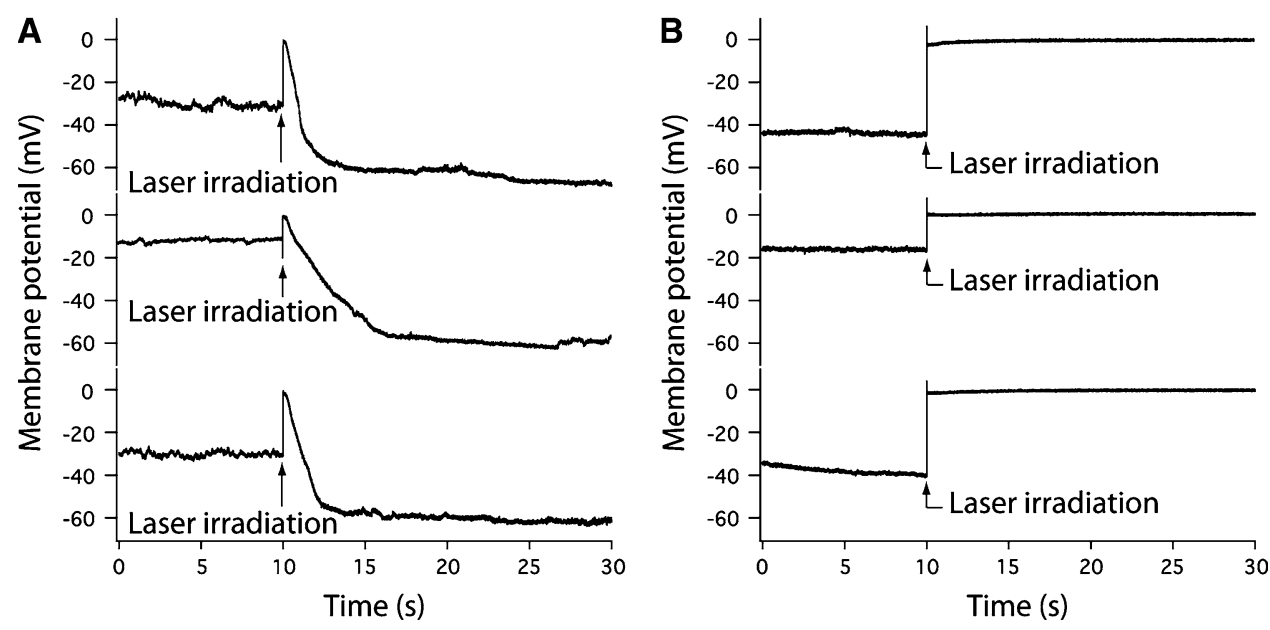
Fig. 5 a Typical behavior of HeLa cell membrane potential following membrane irradiation. b Typical behavior of HeLa cell membrane potential following cytoplasm irradiation. c Comparison of long time course membrane potential monitoring between cytoplasm irradiation and plasma membrane irradiation
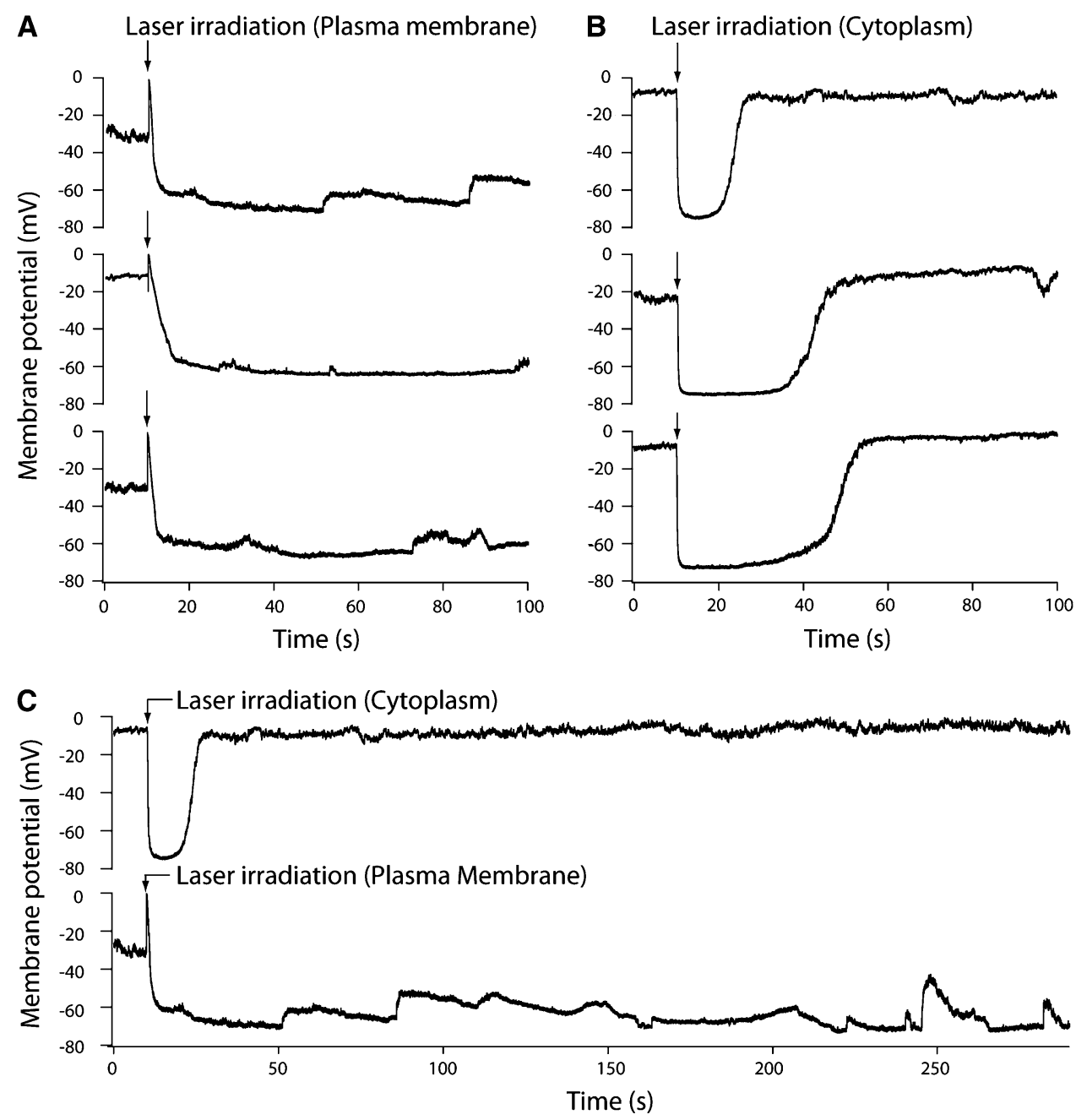

depolarization. Interestingly, membrane potential hyperpolarization after initial depolarization and subsequent recovery follow the same timescale as the $\mathrm{Ca}^{2+}$ level, but not in exact accordance with the $\mathrm{Ca}^{2+}$ level. In contrast, a strong correlation can be observed between the membrane potential and calcium level in the case of cytoplasm irradiation, as shown in Fig. 3. We therefore assume that, for membrane irradiation, the laser caused ion exchange through the membrane, and that in particular, $\mathrm{Ca}^{2+}$ influx can cause repolarization after depolarization through the opening of $\mathrm{Ca}^{2+}$ activated $\mathrm{K}^{+}$channels. Following membrane irradiation (as opposed to cytoplasm irradiation) the membrane voltage behaviour was observed to be unstable and unpredictable, as shown in Fig. 5. Interestingly, the difference in membrane potential behaviour when either the cytoplasm or membrane are irradiated is much more clear than the difference in $\mathrm{Ca}^{2+}$ response. The difference in $\mathrm{Ca}^{2+}$ response following irradiation of either the cytoplasm or membrane was the subject of a previous study (Iwanaga et al. 2006), where various chelating agents and inhibitors were used to isolate the source of the $\mathrm{Ca}^{2+}$ response. In that study, visual inspection alone of the $\mathrm{Ca}^{2+}$ response was insufficient to determine the source of the $\mathrm{Ca}^{2+}$ and the overall properties of the $\mathrm{Ca}^{2+}$ response. By contrast, the membrane potential response measured by patch clamp clearly shows the difference between cytoplasm irradiation and membrane irradiation, as well as giving hints as to the overall viability of the cells. Membrane irradiation appears to more significantly affect the cell condition, as evident by the unstable membrane potential following irradiation. This is a point which should be considered if femtosecond laser irradiation is to be used as a tool for cell stimulation, or for optoporation for transfection of living cells with DNA by femtosecond laser.

In addition, the results in this manuscript also clarify the $\mathrm{Ca}^{2+}$ dye influence on laser induced intracellular $\mathrm{Ca}^{2+}$ dynamics. In 2006, Iwanaga et al. reported on the mechanism of near-infrared femtosecond laser induced $\mathrm{Ca}^{2+}$ release which triggers $\mathrm{Ca}^{2+}$ waves in cells (Iwanaga et al. 2006). The presence of the dye is necessary to measure the $\mathrm{Ca}^{2+}$ dynamics, but does have the potential to affect the laser-cell interaction. In Fig. 2d, by comparing the hyperpolarization power dependence (using no fluorescent dye) and 
Fig. 6 Photogeneration of membrane potential depolarization and $\mathrm{Ca}^{2+}$ waves following laser irradiation of the cell membrane. Upper figures show a time sequence of fluorescence images of HeLa cells stained with the $\mathrm{Ca}^{2+}$ indicator Fluo-4. (White dot shows laser focus, white line shows pipette apex, and white dotted circle shows targeted cell.) Lower figure shows femtosecond laser causing membrane potential repolarization after depolarization and intracellular $\mathrm{Ca}^{2+}$ concentration elevation in HeLa cell. The scale bar is $20 \mu \mathrm{m}$

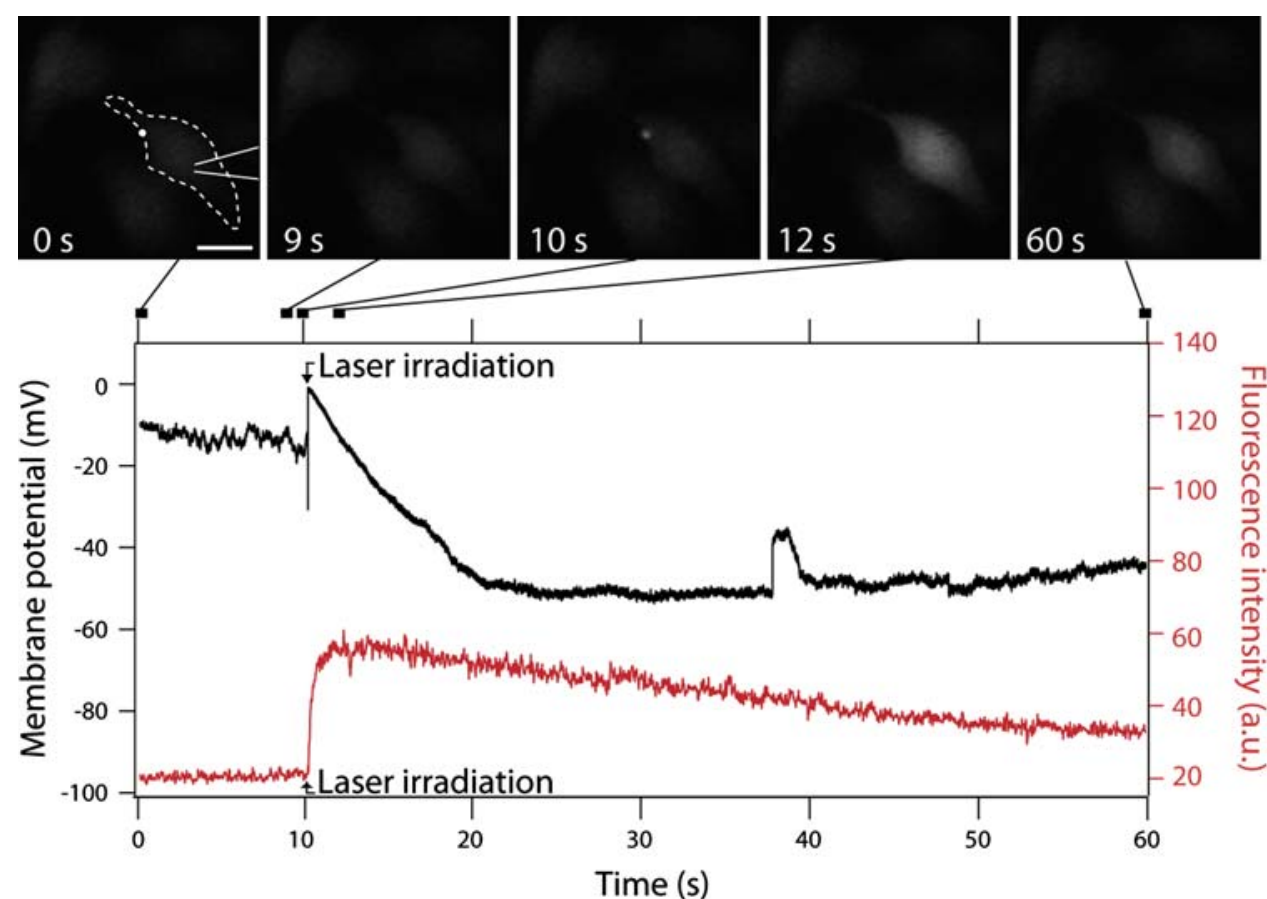

Acknowledgments The authors thank Dr. Hideo Tanaka at the Kyoto Prefectural University of Medicine for technical advice in electrophysiological measurement, and Mr. Tomoya Uchiyama for help with the patch-clamp experimental setup.

Open Access This article is distributed under the terms of the Creative Commons Attribution Noncommercial License which permits any noncommercial use, distribution, and reproduction in any medium, provided the original author(s) and source are credited.

\section{References}

Borle AB, Loveday J (1968) Effects of temperature, potassium, and calcium on the electrical potential difference in HeLa cells. Cancer Res 28:2401-2405

Denk W, Strickler JH, Webb WW (1990) Two-photon laser scanning fluorescence microscopy. Science 248:73-76. doi:10.1126/ science. 2321027

Dombeck DA, Sacconi L, Blanchard-Desce M, Webb WW (2005) Optical recording of fast neuronal membrane potential transients in acute mammalian brain slices by second-harmonic generation Microscopy. J Neurophysiol 94:3628-3636. doi:10.1152/jn. 00416.2005

Fein A, Terasaki M (2005) Rapid increase in plasma membrane chloride permeability during wound resealing in starfish oocytes. J Gen Physiol 126:151-159. doi:10.1085/jgp.200509294

Hirase H, Nikolenko V, Goldberg JH, Yuste R (2002) Multiphoton stimulation of neurons. J Neurobiol 51:237-247. doi:10.1002/neu.10056

Iwanaga S, Kaneko T, Fujita K, Smith N, Nakamura O, Takamatsu T, Kawata S (2006) Location-dependent photogeneration of calcium waves in HeLa Cells. Cell Biochem Biophys 45:167-176. doi: $10.1385 / \mathrm{CBB}: 45: 2: 167$

Iwanage S, Smith N, Fujita K, Kawata S, Nakamura O (2005) Singlepulse cell stimulation with a near-infrared picosecond laser. Appl Phys Lett 87:243901. doi:10.1063/1.2147733

König K, Riemann I, Fischer P, Halbhuber K-J (1999) Intracellular nanosurgery with near infrared femtosecond laser pulses. Cell Mol Biol 45:195-201 
Roy G, Sauvé R (1983) Stable membrane potentials and mechanical K ${ }^{+}$ responses activated by internal $\mathrm{Ca}^{2+}$ in HeLa cells. Can J Physiol Pharmacol 61:144-148

Sacconi L, Tolić-Nørrelykke IM, Antolini R, Pavone FS (2005) Combined intracellular three-dimensional imaging and selective nanosurgery by a nonlinear microscope. J Biomed Opt 10:014002. doi: $10.1117 / 1.1854675$

Sacconi L, Dombeck DA, Webb WW (2006) Overcoming photodamage in second-harmonic generation microscopy: Real-time optical recording of neuronal action potentials. Proc Natl Acad Sci USA 103:3124-3129. doi:10.1073/pnas.0511338103

Sauvé R, Simoneau C, Monette R, Roy G (1986) Single-channel analysis of the potassium permeability in HeLa cancer cells: Evidence for a calcium-activated potassium channel of small unitary conductance. J Membr Biol 92:269-282. doi:10.1007/BF01869395

Smith NI, Fujita K, Kaneko T, Katoh K, Nakamura O, Kawata S, Takamatsu T (2001) Generation of calcium waves in living cells by pulsed-laser-induced photodisruption. Appl Phys Lett 79:1208-1210. doi:10.1063/1.1397255

Smith NI, Kumamoto Y, Iwanaga S, Ando J, Fujita K, Kawata S (2008) A femtosecond laser pacemaker for heart muscle cells. Opt Express 16:8604-8616. doi:10.1364/OE.16.008604

Tilly BC, Tertoolen LFJ, Lambrechts AC, Remorie R, Laat SW, Moolenaar WH (1990) Histamine-H1-receptor-mediated phosphoinositide hydrolysis, $\mathrm{Ca}^{2+}$ signalling and membranepotential oscillations in human HeLa carcinoma cells. Biochem $\mathbf{J}$ 266:235-243

Tirlapur UK, König K (2002) Cell biology: targeted transfection by femtosecond laser. Nature 418:290-291. doi:10.1038/418290a

Uzdensky AB, Savransky VV (1997) Single neuron response to pulseperiodic laser microirradiation. Action spectra and two-photon effect. J Photochem Photobiol B 39:224-228. doi:10.1016/S10111344(97)00013-4

Vogel A, Venugopalan V (2003) Mechanisms of pulsed laser ablation of biological tissues. Chem Rev 103:577-644. doi:10.1021/ cr010379n

Wehner F, Shimizu T, Sabirov R, Okada Y (2003) Hypertonic activation of a non-selective cation conductance in HeLa cells and its contribution to cell volume regulation. FEBS Lett 551:20-24. doi:10.1016/S0014-5793(03)00868-8

Wells J, Kao C, Konrad P, Milner T, Kim J, Mahadevan-Jansen A, Jansen ED (2007) Biophysical mechanism of transient optical stimulation of peripheral nerve. Biophys J 93:2567-2580. doi:10.1529/biophysj.107.104786

Wickson-Ginzburg M, Solomon AK (1981) Electrolyte metabolism in HeLa cells. J Gen Physiol 46:1303-1315 